\title{
Evaluation of the cone-shaped pickup performance for low charge sub-10 fs arrival-time measurements at free electron laser facilities
}

\author{
Aleksandar Angelovski, ${ }^{1, *}$ Michael Kuntzsch, ${ }^{2,3}$ Marie Kristin Czwalinna, ${ }^{4}$ Andreas Penirschke, ${ }_{5}^{1}$ \\ Matthias Hansli, ${ }_{1}^{1}$ Cezary Sydlo, ${ }^{4}$ Vladimir Arsov, ${ }^{5}$ Stephan Hunziker, ${ }^{5}$ Holger Schlarb, ${ }^{4}$ \\ Michael Gensch, ${ }^{2}$ Volker Schlott, ${ }^{5}$ Thomas Weiland, ${ }^{6}$ and Rolf Jakoby ${ }^{1}$ \\ ${ }^{1}$ Technische Universität Darmstadt, Institut für Mikrowellentechnik und Photonik, \\ Merckstrasse 25, 64283 Darmstadt, Germany \\ ${ }^{2}$ Helmholtz-Zentrum Dresden-Rossendorf HZDR, Bautzner Landstrasse 400, 01328 Dresden, Germany \\ ${ }^{3}$ Technische Universität Dresden, Institut für Kern- und Teilchenphysik, \\ Zellescher Weg 19, 01328 Dresden, Germany \\ ${ }^{4}$ Deutsches Elektronen-Synchrotron DESY, Notkestrasse 85, 22607 Hamburg, Germany \\ ${ }^{5}$ Paul Scherrer Institute PSI, 5232 Villigen, Switzerland \\ ${ }^{6}$ Technische Universität Darmstadt, Institut für Theorie Elektromagnetischer Felder, \\ Schlossgartenstrasse 8, 64289 Darmstadt, Germany \\ (Received 17 August 2014; published 30 January 2015)
}

\begin{abstract}
An evaluation of the cone-shaped pickup performance as a part of the high bandwidth bunch arrival-time monitors (BAMs) for a low charge sub-10 fs arrival-time measurements is presented. Three sets of pickups are installed at the free electron laser FLASH at Deutsches Elektronen-Synchrotron, the quasi-cw SRF accelerator ELBE at the Helmholtz-Zentrum Dresden-Rossendorf and the SwissFEL injector test facility at Paul Scherrer Institute. Measurements and simulations are in good agreement and the pickups fulfill the design specifications. Utilizing the high bandwidth BAM with the cone-shaped pickups, an improvement of the signal slope by a factor of 10 is demonstrated at ELBE compared to the BAM with a low bandwidth.
\end{abstract}

DOI: 10.1103/PhysRevSTAB.18.012801

PACS numbers: 29.20.Ej, 41.60.Cr, 41.85.Qg

\section{INTRODUCTION}

In order to study dynamical processes down to a femtosecond (fs) time scale, pump-probe experiments are conducted utilizing ultrashort $\mathrm{x}$-ray pulses from free electron lasers (FELs) $[1,2]$. These time-resolved measurements require synchronization between an external laser (pumping) and the FEL pulse (probing) lower than the pulse duration, i.e., a few femtoseconds. The FEL pulse timing can be determined by a high-resolution arrival-time measurement of the electron bunches at the undulators [3]. A precise measurement of the arrival time improves not only the resolution for the pump-probe experiments, but it is also used for a longitudinal feedback system which stabilizes the electron bunch arrival time at several sections of the machine, and by this reduces the beam arrival-time jitter at the undulators [4-6]. In recent years, the interest of the users for ultrashort $\mathrm{x}$-ray pulses is increasing which requires a low bunch charge ( $20 \mathrm{pC}$ and lower) operation of the accelerator $[7,8]$. Different schemes for bunch arrival time measurements have been implemented so far allowing

\footnotetext{
"angelovski@imp.tu-darmstadt.de

Published by the American Physical Society under the terms of the Creative Commons Attribution 3.0 License. Further distribution of this work must maintain attribution to the author(s) and the published article's title, journal citation, and DOI.
}

for single-shot detection with a few 10 fs resolution and below. Depending on the methods applied they deliver arrival-time information for online monitoring, for active stabilization in feedback systems or for a postprocessing of experimental data. A resonant detection scheme utilizing a radio frequency (rf) phase difference from two single cell phase cavities at $2.805 \mathrm{GHz}$ provides for a sub- $50 \mathrm{fs}$ arrivaltime measurements for bunch charges of $20 \mathrm{pC}$ [9]. This type of monitor is implemented at the Linac Coherent Light Source at Stanford Linear Accelerator Center. Another type of a resonant arrival-time monitor for ultralow charges is designed for the laser-driven wakefield experiments for the relativistic electron gun for atomic exploration (REGAE) [10]. It comprises a $3 \mathrm{GHz}$ cavity pickup with a downconverting $I / Q$ detection scheme used for the laser-to-rf synchronization at REGAE [11]. A cross correlation between the coherent terahertz $(\mathrm{THz})$ radiation from the undulator and an external laser source presented in [12] demonstrated a few femtosecond temporal resolution. A similar scheme utilizes the cross correlation between the x-ray pulses and an external laser with few femtosecond resolution are described in $[13,14]$. Another arrival time detection method is based on THz streaking of the XUV FEL pulse with a THz field generated from an external optical laser. This technique has the potential of a resolution of $10 \mathrm{fs}$ or below [15]. The pulse length and arrival time monitor has recently demonstrated 4 fs arrival-time 
resolution between $\mathrm{x}$-ray and pump laser pulses at SACLA [16].

At the free-electron laser FLASH in Hamburg, pickup based arrival-time monitor with electro-optical detection scheme has been implemented. As an integral part of a laser-based synchronization system, the bunch arrival-time monitors (BAMs) measure the arrival time with a sub- $10 \mathrm{fs}$ time resolution for bunch charges higher than $500 \mathrm{pC}$ [5]. In this scheme, a transient beam-induced signal modulates the amplitude of an external laser pulse in a Mach-Zehnder type electro-optic modulator (EOM). This approximately 200 fs long laser pulse is delivered through a lengthstabilized optical fiber link with a drift stability of around $10 \mathrm{fs} /$ day. Thus, as a direct client of this highly stable optical reference, the BAM based on standard telecom EOMs at $1550 \mathrm{~nm}$ has an intrinsic low drift feature, in addition to the high resolution. The reference timing is the zero crossing of the pickup signal, where the sampling laser pulse has no modulation. The EOM DC bias is such that without an external rf modulation the amplitude of the sampling laser pulses is halved. Any deviation from the zero crossing of the pickup transient, i.e., bunch arrivaltime jitter, results in an amplitude modulation of the reference laser pulse. With a proper calibration with a precession delay line, this amplitude modulation is directly converted to arrival-time information with a dynamic range corresponding to the linear part of the pickup slope. More details are given in [5,17]. The slope steepness at the zero crossing defines the modulation voltage which the laser pulse experiences in the presence of an arrival-time jitter. This determines the time resolution as well as the sensitivity of the BAMs. The slope steepness reduces proportionally with the bunch charge leading to a BAM performance degradation for charges lower than $200 \mathrm{pC}$ [3]. In order to achieve sub-10 fs time resolution for a low charge operation mode (down to $20 \mathrm{pC}$ ), the bandwidth of the current BAMs needs to be increased from the current $12 \mathrm{GHz}$ up to $40 \mathrm{GHz}$ for generating a signal slope higher than $300 \mathrm{mV} / \mathrm{ps}$ [18]. As a part of the high bandwidth BAMs, the cone-shaped pickups were introduced in [19]. The pickup signal has a slope of around $417 \mathrm{mV} / \mathrm{ps}$ and a bandwidth of $40 \mathrm{GHz}$. The pickups have been installed at FLASH (Deutsches ElektronenSynchrotron), at ELBE (Helmholtz-Zentrum DresdenRossendorf) and at the SwissFEL injector test facility (Paul Scherrer Institute).

This paper presents the performance evaluation of the cone-shaped pickups in the three machines using different measurement techniques and instruments. The measurements are compared with the simulation for validation of the performance. Good agreement between measurements and simulations shows the potential of the pickups and the high bandwidth BAM for a sub-10 fs detection of the arrival-time for low charges.

\section{PERFORMANCE EVALUATION AT FLASH, ELBE AND SWISSFEL INJECTOR TEST FACILITY}

The beam-induced transient signal is recorded with an oscilloscope featuring single and combined pickup signals. The measurements are performed directly inside the tunnel at FLASH and ELBE, while at the SwissFEL injector test facility they are conducted outside the tunnel utilizing the electro-optical signal conversion from the BAM system without bandwidth reduction of the measured signal. Two different kinds of oscilloscopes were available for the measurements, a sampling oscilloscope at FLASH and a real time oscilloscope at the other facilities.

\section{A. Measurements at FLASH}

The measurement setup used at FLASH is shown in Fig. 1. Two channels of the sampling oscilloscope Tektronix DSA8300 with 80E10 electrical sampling module and a bandwidth of $50 \mathrm{GHz}$ are connected to the horizontal (combined left and right pickups) and the vertical (single bottom pickup) branch via $40 \mathrm{GHz}$ flexible coaxial cables. The input of the oscilloscope channels is attenuated by $30 \mathrm{~dB}$ for an over voltage protection. The top pickup signal is fed to a $1.3 \mathrm{GHz}$ low pass filter and used as an external trigger. After enabling a stable triggering, the pickup signal wave form is recorded. For an evaluation of the pickup performance, the measurement is compared with the full wave CST PARTICLE STUDIO ${ }^{\circledR}$ and Agilent Advanced Design System (ADS) simulation. The CST PARTICLE STUDIO ${ }^{\circledR}$ wave form is the voltage signal at the output of the pickup for a bunch charge of $20 \mathrm{pC}$. This signal is used as an input in the ADS simulation where the full signal path is simulated using the measured $S$ parameters of the rf components. Figure 2 shows the result of this comparison. The curves are shifted in time in order to have coincident zero crossings. The signal slope at the first zero crossing of the full wave simulation (blue) is $417 \mathrm{mV} / \mathrm{ps}$

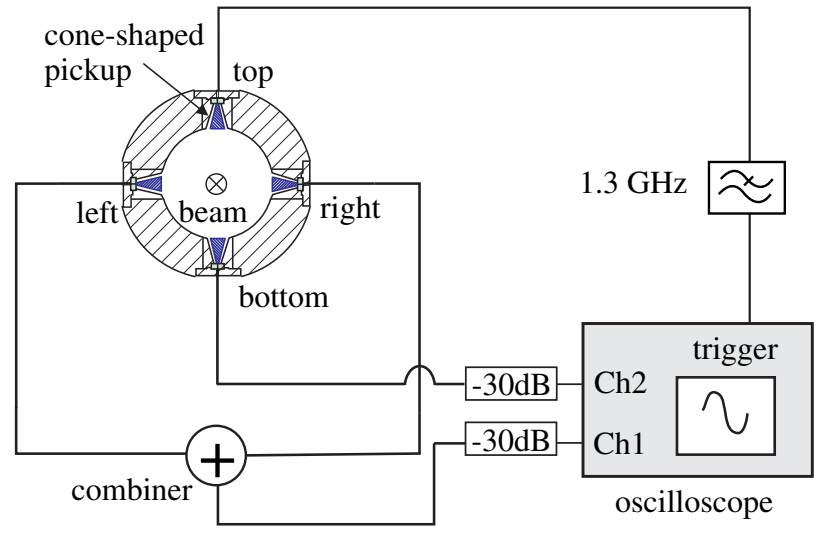

FIG. 1. Measurement setup with the cone-shaped pickups at FLASH. The left and the right pickup are combined and connected to the oscilloscope channel 1, the bottom pickup is connected to channel 2 and the top pickup is used as a trigger. 


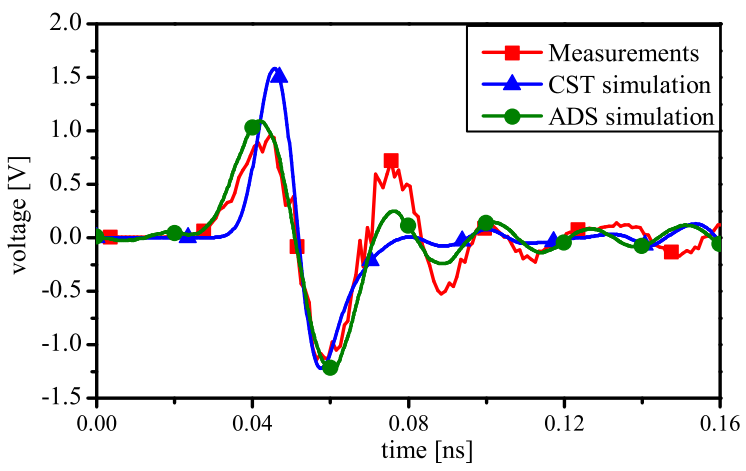

FIG. 2. Comparison of the measured pickup signal with the simulations for a bunch charge of $20 \mathrm{pC}$. The CST simulation shows the signal at the pickup output, while the ADS simulation shows the signal after the rf path.

with a dynamic range defined as the time between the peaks of $13 \mathrm{ps}$. The ADS simulation (green) has a signal slope of $220 \mathrm{mV} / \mathrm{ps}$ and a dynamic range of $18 \mathrm{ps}$. The slope steepness of the measured curve (red) is in between the slope from the ADS simulation and the one from the CST simulation. An exact value is difficult to be extracted due to the measurement uncertainty caused by the unstable triggering. The bunch charge, the shape as well as the arrival time of the bunches fluctuates from bunch to bunch. As the sampling oscilloscope collects samples from several successive wave forms and then builds up a picture of it, the measurement uncertainty caused by the machine fluctuations and the trigger jitter results in kinks and sharp spikes in the recorded pickup signal. The slope reduction as well as the ringing peak around $0.7 \mathrm{ps}$ are due to the signal degradation in the rf path. The obtained results are well inside the specification and meet the pickup requirements for the high bandwidth BAM.

In order to estimate the influence of the bunch position on the signal slope, an orbit scan is performed. The bunch position is measured with a beam position monitor (BPM) directly behind the BAM pickup body. Four steerer magnet coils are used to obtain a parallel displacement with none or negligible tilt angle at the location of the BAM pickups. The pickup signal is recorded at both channels, i.e., single and combined pickup signal. Figure 3 shows the signal slope versus the horizontal and the vertical orbit in case of a single and a combined pickup signal. The horizontal orbit scan [Fig. 3 (top)] shows up to $25 \%$ deviation of the signal slope relative to the centralized beam for both signals within the full range of the beam offset. The deviation is less than $20 \%$ for a beam offset lower than $1 \mathrm{~mm}$. In case of a vertical orbit displacement [Fig. 3 (bottom)], the combined signal shows slope deviation of less than $18 \%$ in the full scan range while the single one has $40 \%$. The slope variation caused by the beam position offset is due to the increasing or decreasing of the peak voltage when the beam is closer or further away from the pickup respectively. For a
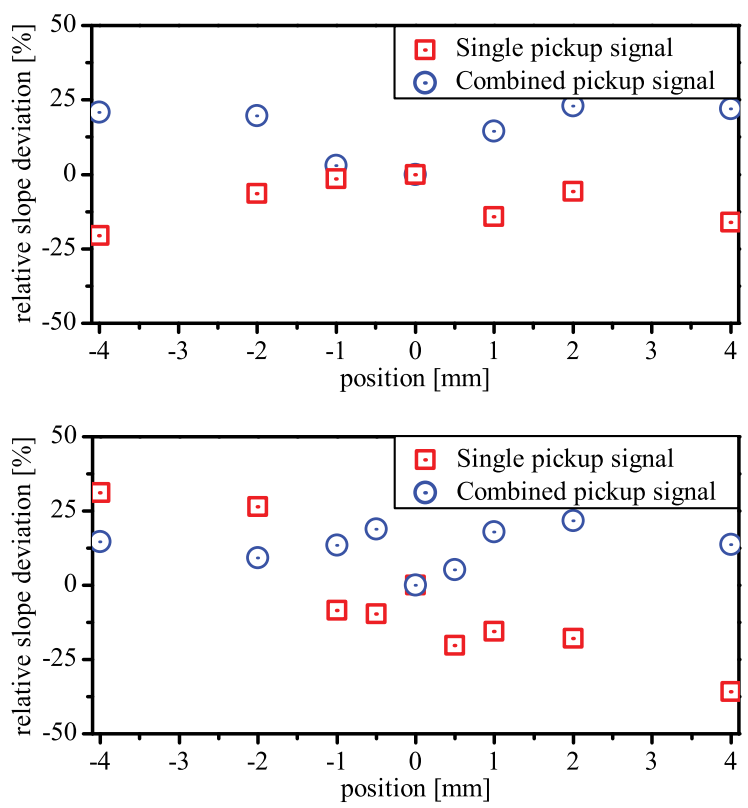

FIG. 3. Signal slope dependence of the orbit change for single and combined signal. Top: Horizontal orbit scan. Bottom: Vertical orbit scan.

single pickup signal the orbit dependence of the slope is more pronounced, unlike for the combined signal where the peak voltage difference is compensated between the opposite pickups. By combining the pickup signal the slope dependence of the orbit is decreased by a factor of 2 , providing for smaller uncertainty in the arrival-time detection. Otherwise, this orbit dependence could lead to a false estimation of the arrival-time by an orbit dependent calibration of the BAM signal. A correction algorithm which utilizes the position readout from the nearby BPM shown in [3] reduces the orbit dependence for the arrivaltime measurements.

\section{B. Measurements at ELBE}

ELBE is a superconducting electron linear accelerator which operates in a quasicontinuous wave (quasi-cw) operation [20]. The injector generates pulses with a maximum repetition rate of $26 \mathrm{MHz}$. A recent upgrade of the accelerator enabled a compression of the bunch length down to a few hundred femtoseconds. The compression scheme of the magnetic chicane generates an arrival-time jitter and influences the bunch stability [17]. Therefore the arrival time of the electron bunches needs to be measured with a femtosecond precision.

The pickups installed at ELBE are measured with a high bandwidth real time oscilloscope Agilent Infiniium 90000 $Q$-Series and with the BAM front-end measurement setup described in [21]. The oscilloscope has two $63 \mathrm{GHz}$ real time channels with a sampling rate of $160 \mathrm{GS} / \mathrm{s}$ and provides for the possibility to record single and/or multiple shots of the signal wave form. The multiple shots feature 


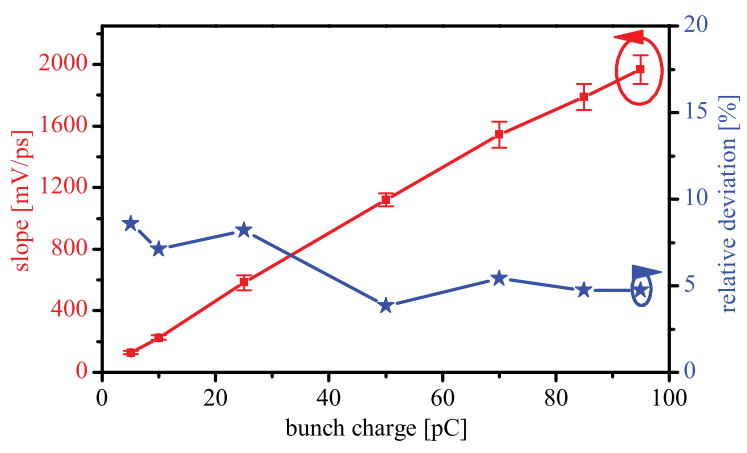

FIG. 4. Pickup signal slope dependence of the bunch charge. The blue curve shows the relative slope deviation for 128 shots.

allows the study of the time domain pickup signal behavior from one shot to the other. The measurement setup is similar to the one in Fig. 1 with the oscilloscope installed inside the beam line tunnel. However, as an external triggering is not necessary for this measurement, the top port of the pickup is terminated with a matched load. Figure 4 shows the signal slope for a bunch charge sweep from $5 \mathrm{pC}$ up to $95 \mathrm{pC}$. The measured signal slope for bunch charge of $20 \mathrm{pC}$ is around $360 \mathrm{mV} / \mathrm{ps}$ and corresponds to the simulation as well as to the measurements at FLASH (see Fig. 2). The uncertainty, i.e., the absolute noise level, is obtained from a set of 128 consecutive shots and it is increasing as the bunch charge increases. The relative deviation which is a combination of the relative precision of the detection and the bunch charge fluctuation remains below $10 \%$ for the entire range of the bunch charge sweep.

In order to compare the performance of the high $(40 \mathrm{GHz})$ and the low $(12 \mathrm{GHz})$ bandwidth BAM, the data acquisition was done utilizing the BAM front end. An EOM with a bandwidth up to $35 \mathrm{GHz}$ as a part of the high and an EOM up to $12 \mathrm{GHz}$ as a part of the low bandwidth BAM is used for the measurements. The signal from the cone-shaped pickups modulates the laser pulse train in the EOM. The optical output is converted to an electrical signal with a photodiode and is fed into a 16 bit Analog-to-digital converter (ADC). The third harmonic from a photodiode, fed with the laser reference pulse train $(3 \times 78 \mathrm{MHz})$ is used to clock the ADC. The data from the ADC is processed with a Field-programmable gate array giving the baseline value and the modulation depth for every bunch [21]. The output is the mean value of $200 \mathrm{k}$ measured points in order to reduce the influence of the fast jitter. Knowing the half-wave voltage $V_{\pi}$ of the EOM and the cable losses, the absolute pickup signal voltage is calculated. Figure 5 shows the comparison between the pickup signal measured with the oscilloscope (blue), the high $35 \mathrm{GHz}$ EOM (red) and the low bandwidth $12 \mathrm{GHz}$ EOM (black) BAM front end. The oscilloscope curve and the high bandwidth BAM output signal are in a good agreement. The cutting in the negative part of the EOM curve is

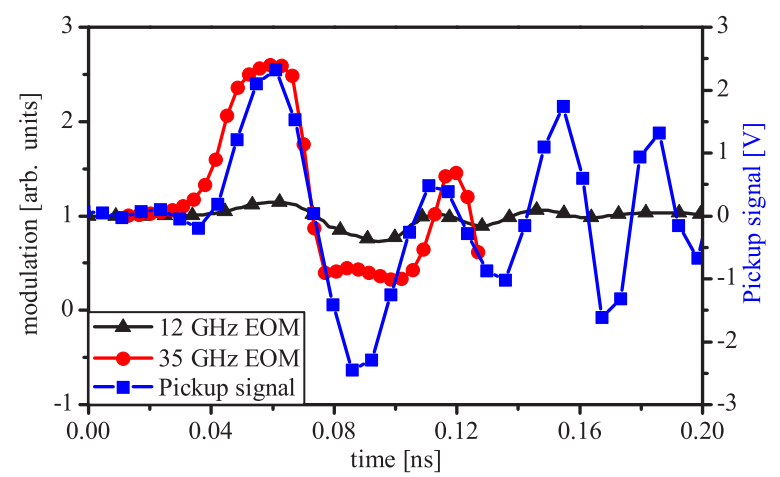

FIG. 5. Comparison between the cone-shaped pickup signal measured with a real time oscilloscope (blue curve), with a high bandwidth (red curve) and state of the art (black curve) BAM front end for a bunch charge of $80 \mathrm{pC}$.

due to the limited extinction ratio of the used EOM. This does not affect the BAM sensitivity as it operates in the region around the zero crossing. However, it reduces the dynamic range of the BAM and using an EOM with a higher extinction ratio will minimize this effect. The ringing which appears after the beam-induced signal is due to the wakefields, which are caused by the beam pipe abrupt diameter change for the $\mathrm{THz}$ setup, only a few centimeters before the pickup. In comparison to the current low bandwidth BAM front end, the high bandwidth system improves the slope by a factor of 10 and it has the potential to measure the arrival time with a few femtosecond time resolution for low charges.

\section{Measurements at the SwissFEL injector test facility}

Unlike the measurements done at FLASH and ELBE, the measurements at the SwissFEL injector test facility are conducted outside the tunnel without loss of bandwidth of the measured pickup signal. Figure 6 shows the schematic of the measurement setup. The beam-induced pickup signal is connected to the rf input of the EOM via a power limiter in the BAM box. A cw laser signal with $1550 \mathrm{~nm}$ wavelength is transported through the usual single-mode optical fiber link-BAM-photoreceiver chain-and is fed into the

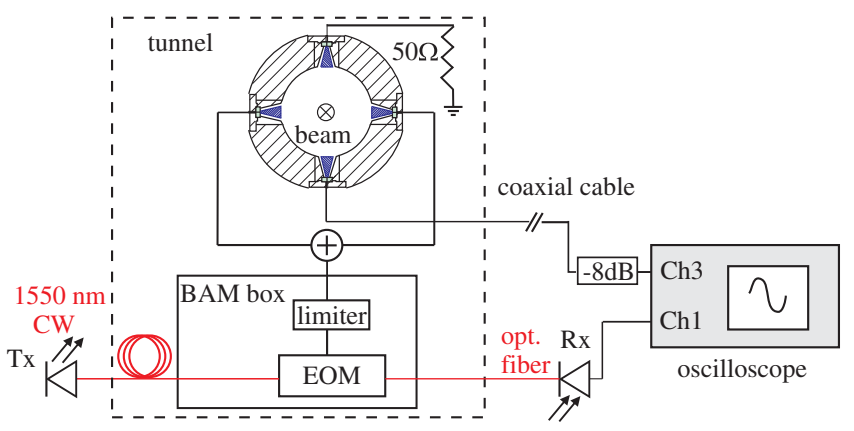

FIG. 6. Measurement setup for the pickup signal extraction utilizing the BAM front end and a real time oscilloscope. 


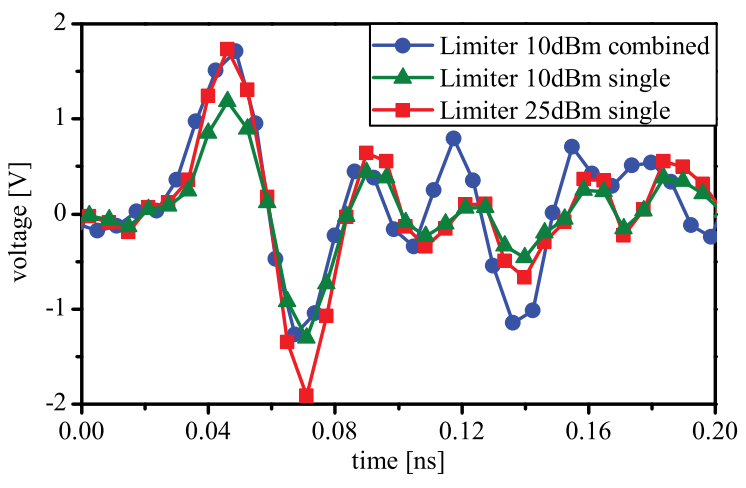

FIG. 7. Comparison of the measured beam-induced pickup signal. A single and a combined pickup signal is compared with two types of limiters.

optical input of the EOM where it is modulated by the pickup signal. The modulated optical signal is transported via optical fiber to a photodiode type XPDV2120 $(50 \mathrm{GHz}$, $u 2 t$ ) outside the tunnel where the output is recorded with the real time oscilloscope (the same as in ELBE). In order to protect the EOM from an over voltage damage in case of high charges, a power limiter is used at the rf input. The limiting threshold and the bandwidth of the limiter influence the pickup signal characteristics, i.e., the modulation signal for the EOM. In order to estimate the influence, two sets of measurements are conducted with different types of limiters. The first limiter (Agilent N9355F) has a cutoff frequency of $50 \mathrm{GHz}$ and a limiting threshold of $10 \mathrm{dBm}$, while the second one (Agilent N9355C) has a cutoff frequency of $26.5 \mathrm{GHz}$ and a limiting threshold of $25 \mathrm{dBm}$. Figure 7 shows the comparison between a single pickup and a combined pickup signal when using the different types of limiters for a bunch charge of $40 \mathrm{pC}$.

The signal slope is almost identical in the case of a single pickup with higher $(25 \mathrm{dBm})$ and the combined signal with lower $(10 \mathrm{dBm})$ limiting threshold. It is around $10 \%$ lower compared to the slope measured at FLASH and ELBE due to losses in the signal path, but it is still within the specifications. For the single pickup signal with a $10 \mathrm{dBm}$ limiter the slope steepness is decreased compared to the other curves. The higher limiting threshold provides for a higher peak voltage which leads to a steeper slope even though the bandwidth of the limiter is smaller compared to the other one. The dynamic range of the measured pickup signal is around $20 \mathrm{ps}$ and it is higher compared to the one measured at FLASH (18 ps) with the sampling oscilloscope. This can be attributed to signal degradation in the signal path as well as to the lower number of measurement points provided by the real time oscilloscope compared to the sampling one.

\section{SUMMARY}

The cone-shaped pickups proposed in [19], as a part of the high bandwidth BAM for low charge sub-10 fs arrival-time detection have been installed at FLASH, ELBE and the Swiss FEL injector test facility. An evaluation of the pickups is performed at the three facilities utilizing high bandwidth oscilloscopes and the BAM front end. The measurements are in good agreement with the simulations. The pickup signal has a slope of around $360 \mathrm{mV} / \mathrm{ps}$ for $20 \mathrm{pC}$ and fulfills the design specification for all three accelerators. By combining the pickup signals the orbit dependence of the signal slope can be reduced by a factor of 2. As shown at ELBE, the signal obtained from the high bandwidth BAM utilizing the cone-shaped pickups has 10 times higher slope steepness compared to a low bandwidth BAM, demonstrating the potential for few femtosecond arrival-time measurements at low charges.

\section{ACKNOWLEDGMENTS}

The authors would like to thank the company CST for providing the CST STUDIO SUITE® software package. For providing the real time $63 \mathrm{GHz}$ oscilloscope Agilent Infiniium $90000 Q$-Series and the technical support, the authors would like to thank Mr. Andreas Siegert and Dr. Thomas Kirchner from Agilent Technologies Sales \& Services $\mathrm{GmbH} \&$ Co. KG. The work of A. Angelovski is supported by the German Federal Ministry of Education and Research (BMBF) within Joint Project No. FSP 302.

[1] A. Azima, S. Dsterer, P. Radcliffe, H. Redlin, N. Stojanovic, W. Li, H. Schlarb, J. Feldhaus, D. Cubaynes, M. Meyer et al., Appl. Phys. Lett. 94, 144102 (2009).

[2] H. Redlin, A. Al-Shemmary, A. Azima, N. Stojanovic, F. Tavella, I. Will, and S. Dsterer, Nucl. Instrum. Methods Phys. Res., Sect. A 635, S88 (2011).

[3] M. K. Bock, Ph.D. thesis, Universität Hamburg, 2012.

[4] P. Craievich, S. Di Mitri, M. Milloch, G. Penco, and F. Rossi, Phys. Rev. ST Accel. Beams 16, 090401 (2013).

[5] F. Löhl, V. Arsov, M. Felber, K. Hacker, W. Jalmuzna, B. Lorbeer, F. Ludwig, K.-H. Matthiesen, H. Schlarb, B. Schmidt et al., Phys. Rev. Lett. 104, 144801 (2010).

[6] C. Schmidt, M. K. Bock, S. Pfeiffer, H. Schlarb, W. Koprek, and W. Jalmuzna, in Proceedings of FEL2011, Shanghai, China, 2011 (JACoW, Geneva, 2011), pp. 531534.

[7] X. Wang and X. Chang, Nucl. Instrum. Methods Phys. Res., Sect. A 507, 310 (2003).

[8] J. Rosenzweig, D. Alesini, G. Andonian, M. Boscolo, M. Dunning, L. Faillace, M. Ferrario, A. Fukusawa, L. Giannessi, E. Hemsing et al., Nucl. Instrum. Methods Phys. Res., Sect. A 593, 39 (2008).

[9] A. Brachmann et al., in Proceedings of the International Particle Accelerator Conference, Kyoto, Japan (ICR, Kyoto, 2010), pp. 2287-2289.

[10] M. Hansli, A. Angelovski, A. Penirschke, F. J. Gruener, B. Zeitler, K. Floettmann, D. Lipka, H. Schlarb, S. C. Vilcins, and R. Jakoby, in Proceedings of IPAC 2014, 
Dresden, Germany, 2014 (JACoW, Geneva, 2014), pp. 1820-1822.

[11] M. Felber, M. Hoffmann, U. Mavric, H. Schlarb, S. Schulz, and W. Jalmuzna, in Proceedings of the 3rd International Particle Accelerator Conference, New Orleans, LA, 2012 (IEEE, Piscataway, NJ, 2012), pp. 2624-2626.

[12] F. Tavella, N. Stojanovic, G. Geloni, and M. Gensch, Nat. Photonics 5, 162 (2011).

[13] M. Harmand, R. Coffee, M. R. Bionta, M. Chollet, M. French, D. Zhu, D. M. Fritz, H. T. Lemke, N. Medvedev, B. Ziaja et al., Nat. Photonics 7, 215 (2013).

[14] C. Gahl, A. Azima, M. Beye, M. Deppe, K. Dobrich, U. Hasslinger, F. Hennies, A. Melnikov, M. Nagasono, A. Pietzsch et al., Nat. Photonics 2, 165 (2008).

[15] I. Grguras, A. R. Maier, C. Behrens, T. Mazza, T. J. Kelly, P. Radcliffe, S. Dusterer, A. K. Kazansky, N. M. Kabachnik, T. Tschentscher et al., Nat. Photonics 6, 852 (2012).
[16] P. N. Juranić et al. (unpublished).

[17] F. Löhl, Ph.D. thesis, Universität Hamburg, 2009.

[18] K. Hacker, Ph.D. thesis, Universität Hamburg, 2010.

[19] A. Angelovski, A. Kuhl, M. Hansli, A. Penirschke, S. M. Schnepp, M. Bousonville, H. Schlarb, M. K. Bock, T. Weiland, and R. Jakoby, Phys. Rev. ST Accel. Beams 15, 112803 (2012).

[20] F. Gabriel, P. Gippner, E. Grosse, D. Janssen, P. Michel, H. Prade, A. Schamlott, W. Seidel, A. Wolf, and R. Wnsch, Nucl. Instrum. Methods Phys. Res., Sect. B 161-163, 1143 (2000).

[21] M. Kuntzsch, M. Gensch, U. Lehnert, F. Roeser, R. Schurig, M. Bousonville, M. K. Czwalinna, H. Schlarb, S. Schulz, and S. Vilcins, in Proceedings of the 4th International Particle Accelerator Conference, IPAC2013, Shanghai, China, 2013 (JACoW, Geneva, 2013), pp. 2932-2934. 